\title{
Extremum principles for biological continuous bodies undergoing volumetric and surface growth
}

\author{
J.F. GANGHOFFER* \\ Lemta - Ensem, 2 Avenue de la Forêt de Haye, BP 160, 54504 Vandoeuvre Cedex, France
}

\begin{abstract}
The volumetric and surface growth of continuum solid bodies is considered, in the framework of the thermodynamics of open systems exchanging mass, work and chemical species (nutrients) with their environment. More specifically, we address the issue of setting up extremum principles for such growing bodies. A general three-field variational principle is set up, based on the so-called zero potential, which is a byproduct of the grand potential. The stationnarity conditions of those potentials deliver balance laws for generalized volumetric and surface Eshelby tensors, leading further to the identification of the material forces for growth.
\end{abstract}

Key words: thermodynamics of open systems, extreme principles, Eshelby tensors, volumetric growth, surface growth.

\section{Introduction}

Growth of biological tissues has attracted the attention of several researchers in continuum mechanics within the last two decades [1-9]. The most advanced works treat the interactions between the mechanical equilibrium and the transport phenomena leading to growth, see $[10,11]$ using the theory of mixtures, or [12] highlighting the coupling between reaction-transport of solutes and mechanics.

While most of the efforts have been spent on the modeling of volumetric growth, fewer works deal with surface growth, which from a conceptual point of view introduces the additional difficulty of a changing number of particles, in comparison with volumetric growth (an assumption of constant particle numbers is made there, considering that either the density or local volume do change instead). A unifying framework for the treatment of both bulk and surface growth phenomena has been proposed in [13], relying on the introduction of configurational forces as the internal driving forces for growth. Configurational forces for surface growth have been identified in [14], with application to bone remodeling.

While most of the growth models are devoted to writing evolution laws for kinematic like variables related to the growth process (growth tensor), the generalization of variational principles to account for the multiphysical phenomena occurring during growth has not been achieved so far. The main objective of this contribution is then to set up extremum principles for continuum solid bodes submitted to growth, considering successively volumetric and surface growth. This last situation differs from volumetric growth by the consideration of a specific material behavior of the growing surface.

Since biological tissues are open systems exchanging nutrients with their environment, it is quite natural to adopt the umbrella of the thermodynamics of open systems, allowing incorporating the various kinds of energies growing bodies are exchanging. We thereby advocate a novel contribution, especially the writing of new extremum principles for growing solid bodies involving generalized Eshelby stresses. This will extend the writing of nonlinear elasticity extremum principles involving the standard mechanical Eshelby stress [17]. Regarding notations, vectors and second order tensors will be denoted as boldface symbols.

\section{Extremum principle for volumetric growth}

We consider a representative tissue element (chosen at least one order of magnitude larger than the typical size of the heterogeneities, and at least one order of magnitude smaller than the macroscopic size of the body) as an open heterogeneous system exchanging work, mass and heat with its surrounding. The field variables are the absolute temperature $\theta(\mathbf{x}, t)$, the entropy density $s(\mathbf{x}, t)$, the transformation gradient $\mathbf{F}(\mathbf{x}, t)$, the chemical potentials $\left\{\mu_{k}(\mathbf{x}, t)\right\}_{k=1 . . N}$ and the number of moles (densities) of the $N$ chemical species (nutrients) $\left\{n_{k}(\mathbf{x}, t)\right\}_{k=1 . . N}$. The position $\mathbf{x}$ is introduced as a label of the material particles which occupy a finite volume in space.

As a starting point, the grand potential, a quantity used in statistical mechanics particularly for irreversible processes occurring in continuous open systems [15], is introduced in terms of its spatial density

$$
\begin{gathered}
\omega_{v o l}=\omega_{v o l}\left(\theta(x, t), \mathbf{F}(x, t), \mu_{k}(x, t)\right) \\
:=\int \mathbf{T}: d \mathbf{F}+\int \mathbf{F}: d \mathbf{T}
\end{gathered}
$$

$\omega_{v o l}$ is the sum of the strain energy density

$$
W_{0}=W_{0}(\mathbf{F}):=\int \mathbf{T}: d \mathbf{F}
$$

and the complementary strain energy density

$$
W_{0}^{c}(\mathbf{T}):=\int \mathbf{F}: d \mathbf{T},
$$

\footnotetext{
*e-mail: jean-francois.Ganghoffer@ensem.inpl-nancy.fr
} 
J.F. Ganghoffer

with $\mathbf{T}:=\partial_{F} \omega$ the first Piola-Kirchhoff stress (the listed arguments of $\omega_{v o l}$ will be justified later on). The previously defined densities $W_{0}(\mathbf{F})$ and $W_{0}^{c}(\mathbf{T})$ represent the area below and above the curve $\mathbf{T}=\mathbf{T}(\mathbf{F})$ respectively; hence, $\omega_{v o l}$ expresses as

$$
\omega_{v o l}\left[\theta, \mathbf{F}, \mu_{k}\right]=\mathbf{T}: \mathbf{F}
$$

assuming the existence of a strain and stress free reference state.

Introducing next the internal energy density $e\left(\mathbf{F}, s, n_{k}\right)$, the generalized fundamental Euler's relation traducing the homogeneity of degree one of $e\left(\mathbf{F}, s, n_{k}\right)$ writes as [16]

$$
\begin{gathered}
e\left(\mathbf{F}, s, n_{k}\right)=\mathrm{T}\left(\mathbf{F}, s, n_{k}\right): \\
\mathbf{F}+\theta\left(\mathbf{F}, s, n_{k}\right) s-\mu_{k}\left(\mathbf{F}, s, n_{k}\right) n_{k} .
\end{gathered}
$$

Observe that the mechanical contribution of the internal energy density, viz the scalar $\mathrm{T}\left(\mathbf{F}, s, n_{k}\right): \mathbf{F}$, incorporates both the strain energy and the complementary strain energy densities.

Consideration of (3) then leads to the rewriting of (2) as

$$
\begin{gathered}
\omega_{v o l}[\theta, \mathbf{F}, \mu]=e(\mathbf{F}, s, \mathbf{n})-\theta(\mathbf{F}, s, \mathbf{n}) s \\
-\mu_{k}(\mathbf{F}, s, \mathbf{n}) \cdot n_{k}
\end{gathered}
$$

in which the convention of summation of the repeated index is adopted.

From the Gibbs and Gibbs-Duhem relations and using (4), the differential of the grand potential is obtained from a straightforward calculation

$$
\begin{aligned}
d \omega_{v o l} & =-s d \theta+\mathbf{T}: d \mathbf{F}-n_{k} d \mu_{k} \\
& \equiv \mathbf{T}: d \mathbf{F}+\mathbf{F}: d \mathbf{T}
\end{aligned}
$$

resulting in the thermodynamic relations

$$
\begin{gathered}
\frac{\partial \omega_{v o l}}{\partial \theta}=-s ; \quad \frac{\partial \omega_{v o l}}{\partial \mathbf{F}}=\mathbf{T} ; \\
\frac{\partial \omega_{v o l}}{\partial \mu_{k}}=-n_{k}
\end{gathered}
$$

highlighting $\theta, \mathbf{F}, \mu_{k}$ as the arguments of $\omega_{v o l}$; those constitutive like relations shall be called state laws in the sequel. The contributions on the r.h.s. of $d \omega_{v o l}$ in (5) are successively the quasi-static heat flux, the incremental work of the internal stresses and the so-called quasi-static chemical work [16].

A variational principle for volumetric growth is next constructed. From the static equilibrium equation of the tissue element submitted to body forces $\mathbf{f}_{0}$

$$
\nabla_{X} \cdot \mathbf{T}+\mathbf{f}_{0}=\mathbf{0}
$$

the definition of the transformation gradient $\mathbf{F}:=\nabla_{X} \mathbf{x}$, and an integration by part, a weak formulation linking the integral of $\omega$ on a control volume $V$ to contributions involving the applied loading (body forces and surface tractions T.N) is obtained in a straightforward manner:

$$
\int_{V} \omega_{v o l} d V=\int_{V} \mathbf{f}_{0} \cdot \psi d V+\int_{\partial V}(\mathbf{T} \cdot \mathbf{N}) \cdot \psi d S
$$

with $\psi$ a kinematically admissible position field. The boundary $\partial V$ is partitioned into a subpart $S_{\psi}$ with imposed placement $\psi^{d}$ and a complementary part $S_{t}$ with imposed tractions $\mathbf{T}^{d}$, according to

$$
\begin{gathered}
\int_{V} \omega_{v o l} d V=\int_{V} \mathbf{T}: \mathbf{F} d V \\
=\left\{\int_{V} \mathbf{f}_{0} \cdot \psi d V+\int_{S_{t}}\left(\mathbf{T}^{d} \cdot \mathbf{N}\right) \cdot \psi d S\right\} \\
+\int_{S_{\psi}}(\mathbf{T} \cdot \mathbf{N}) \cdot \psi^{d} d S \equiv W_{T}+W_{\psi}
\end{gathered}
$$

introducing therein the works of the imposed body forces and tractions $W_{T}$ and the work of the imposed placements, viz the quantity $W_{\psi}=\int_{S_{\psi}}(\mathbf{T} . \mathbf{N}) \cdot \psi^{d} d S$.

The Gibbs-Duhem relation allows expressing one of the intensive variables in the triplet $\left(\theta, \mathbf{T}, \mu_{k}\right)$ vs. the two remaining variables. Using (8), a Legendre transform of $\omega$ versus the work terms gives a new potential, which identically vanishes, called for this reason the zero potential, elaborated as

$$
\begin{gathered}
\Omega\left[\theta, \psi, \mu_{k}\right]:=\int_{V} \omega_{v o l} d V \\
-\left\{\int_{V} \mathbf{f}_{0} \cdot \psi d V+\int_{S_{t}}\left(\mathbf{T}^{d} \cdot \mathbf{N}\right) \cdot \psi d S\right\} \\
-\int_{S_{\psi}}(\mathbf{T} \cdot \mathbf{N}) \cdot \psi^{d} d S \equiv 0 .
\end{gathered}
$$

Observe that the zero potential constructed thereabove is the tensorial generalization of the zero potential [15], accounting for the boundary conditions over the tissue element.

Since the zero potential is nil, it enjoys the property of extremality

$$
\delta \Omega\left[\theta, \psi, \mu_{k}\right]=0
$$

for the solution field, the set of primary variables $\left(\theta, \psi, \mu_{k}\right)$ at thermodynamic equilibrium; this condition holds whatever the state laws is. Thereby, a generalized potential energy in a thermodynamic framework has been set up, allowing for the consideration of the thermal and chemical forms of energy, in addition to the mechanical energy.

It is worth noting that the stress has been substituted by the temperature and chemical potential as new control variables: it is thus not possible to control the stress, and essential boundary conditions on the portion of surface $S_{t}$ have to be prescribed in the formulation of the associated extremum principle, namely

$$
\mathbf{T} . \mathbf{N}=t^{d} \quad \text { on } \quad S_{0 t} .
$$

The material variation of the potential $\Omega\left[\theta, \psi, \mu_{k}\right]$ is next calculated to express the stationnarity condition (at fixed material position: the index $X$ is dropped in the variation, hence $\left.\delta \equiv \delta_{X}\right)$ following the steps: 
1. The material variation of $\omega$ is formulated as

$$
\begin{gathered}
\delta \omega_{v o l}=\delta \omega_{v o l}\left(\theta, \mathbf{F}=\nabla_{X} \psi, \mu_{k}\right) \\
\equiv \frac{\partial \omega_{v o l}}{\partial \nabla_{X} \psi}: \delta\left(\nabla_{X} \psi\right)+\frac{\partial \omega_{v o l}}{\partial \theta} \delta \theta+\frac{\partial \omega_{v o l}}{\partial \mu_{k}} \delta \mu_{k} .
\end{gathered}
$$

2. The first contribution on the right hand side is integrated by part in the corresponding integral term in $\Omega\left[\theta, \psi, \mu_{k}\right]$, by a standard calculation in configurational mechanics, thereby highlighting the Eshelby stress built from the grand potential, defined as

$$
\Sigma:=\omega_{v o l} \mathbf{I}-\mathbf{F}^{T} \cdot \mathbf{T}
$$

3. The variations of the spatial position, temperature and chemical potential in previous expression are further expressed as

$$
\delta_{x} \psi+\mathbf{F} . \delta_{x} \mathbf{X}=\mathbf{0} ; \quad \delta \theta=\nabla_{X} \theta . \delta \mathbf{X} ; \quad \delta \mu_{k}=\nabla_{X} \mu_{k} . \delta \mathbf{X}
$$

Assembling the calculations in those three steps delivers the material variation of the zero potential

$$
\begin{gathered}
\delta \Omega\left[\theta, \psi, \mu_{k}\right]=\int_{V} \operatorname{Div}\left(-\Sigma-\left(s \theta+n_{k} \mu_{k}\right) \mathbf{I}\right) \cdot \delta \mathbf{X} d X \\
-\int_{V}\left(\theta \operatorname{Div}(s \mathbf{I})+\mu_{k} \operatorname{Div}\left(n_{k} \mathbf{I}\right)\right) \cdot \delta \mathbf{X} d X \\
+\int_{V}\left(\partial_{X} W_{0}\right)_{\exp l} \cdot \delta \mathbf{X} d X+\int_{V} \mathbf{F}^{\mathbf{T}} \cdot \mathbf{f}_{\mathbf{0}} \cdot \delta \mathbf{X} d X \\
+\int_{S_{t}} \frac{\partial \omega}{\partial \nabla_{X} \psi} \cdot \mathbf{N} \cdot \delta \psi d S-\int_{S_{t}} \mathbf{t}^{d} \cdot \delta \psi d S \\
-\int_{S_{\psi}} \delta(\mathbf{T} \cdot \mathbf{N}) \cdot \psi^{d} d S .
\end{gathered}
$$

The Eshelby stress defined in (14) and involved in (15) incorporates the density of the grand potential, sum of the strain energy density $W_{0}(\mathbf{F})$ and of the complementary energy density $W_{c}(\mathbf{T})$; it thus differs from the classical version, which relies on $W_{0}(\mathbf{F})$ exclusively. One should note that the complementary energy density should ideally be based on true tensors (like the second Piola-Kirchhoff stress and its conjugated strain measure in the sense of energy).

The volumetric contribution in (15) is then identified as

$$
\begin{aligned}
\delta \Omega_{v o l}=0 \Rightarrow & \operatorname{Div} \Xi+\left(\theta \nabla_{X} s+\mu_{k} \nabla_{X} n_{k}\right) \\
& +\mathbf{F}^{T} \cdot \mathbf{f}_{0}=\mathbf{0}
\end{aligned}
$$

introducing therein the following energy momentum tensor in a thermodynamic context

$$
\Xi:=-\Sigma-\left(s \theta+n_{k} \mu_{k}\right) \mathbf{I} .
$$

This energy momentum tensor incorporates all forms of energies (mechanical through the classical Eshelby stress [17] $\Sigma:=\mathbf{F}^{T} . \mathbf{T}-W \mathbf{I}$, and thermal and chemical forms in the last isotropic contribution), in line with $[18,19]$ who introduced more general internal variables in place of the chemical variables. Expressions of various energy momentum tensors including the so-called strain energy per unit stress-free volume (at fixed temperature and molar concentrations), have been proposed, see ([20, 21], Eqs. (45), (46)); the reader is also referred to the pioneering work [22] related to those tensors.

The balance law (16) is supplemented by the natural boundary conditions

$$
t^{d}=\frac{\partial \omega}{\partial \nabla_{X} \psi} \cdot \mathbf{N}
$$

Summarizing, a complete model of volumetric growth has been elaborated, formalized as the closed set of equilibrium equations (16) with boundary conditions (18), and constitutive behavior formally given by the dependency of the spatial density of the grand potential $\omega_{v o l}$ versus its arguments, as written in the state laws (6). This thermodynamic framework is further extended to account for situations in which surface growth is of importance, in addition to volumetric growth.

\section{Thermodynamic potential for surface and bulk growth and extremum principle}

Independently from the volumetric contribution, one shall also consider an additional surface potential with (surface) density $\omega_{\text {surf }}[\widetilde{\theta}, \widetilde{\mathbf{F}}, \widetilde{\mu}]$; this last contribution is the surface grand potential and is identified from the superficial excess quantity associated to $d \omega_{\text {surf }}$, viz

$$
d \omega_{\text {surf }} \equiv d \omega^{\sigma}=-s^{\sigma} d \theta-n_{k}^{\sigma} d \mu_{k}+\widetilde{\mathbf{T}}^{e l}: d \widetilde{\mathbf{F}} .
$$

It involves surface elastic stresses and conjugated strains, denoted as the quantities $\widetilde{\mathbf{T}}^{e l}, \widetilde{\mathbf{F}}$ respectively. Gibbs (1928) point of view of excess quantities at the interface between two phases has herewith been adopted (there is in Gibbs point of view no extra volume of the interface separating the two phases). The surface excess

$$
\omega^{\sigma}=\widetilde{\mathbf{T}}^{e l}: \widetilde{\mathbf{F}}
$$

is the surface counterpart of (2). Equality (19) shows that the true thermodynamic arguments of $\omega_{\text {surf }}$ are the (surface temperature), the superficial chemical potential and the surface strain. This last quantity may be chosen as an independent field or as the surface projection of $\mathbf{F}$.

The integration over a control volume (control surface resp.) of the densities $\omega_{\text {vol }}[\theta, \mathbf{F}, \mu]\left(\omega_{\text {surf }}[\widetilde{\theta}, \widetilde{\mathbf{F}}, \widetilde{\mu}]\right.$ resp.) further satisfies the identity

$$
\begin{gathered}
\int_{\Omega_{g}} \omega_{v o l}[\theta, \mathbf{F}, \mu] d X_{g}+\int_{S_{g}} \omega_{\text {surf }}[\widetilde{\theta}, \widetilde{\mathbf{F}}, \widetilde{\mu}] d \sigma_{g} \\
=\int_{\Omega_{g}} \mathbf{T}: \mathbf{F} d X_{g}+\int_{S_{g}} \widetilde{\mathbf{T}}^{e l}: \widetilde{\mathbf{F}} d \sigma_{g}
\end{gathered}
$$

fully expressed in the reference configuration. The right-hand side of previous equality involves the strain energy and complementary strain energy (they sum up to $\mathbf{T}: \mathbf{F}$ and $\widetilde{\mathbf{T}}^{e l}: \widetilde{\mathbf{F}}$ regarding the volumetric and surface contributions respectively) for both the bulk and the surface.

The Gibbs adsorption equation may further be written

$$
S^{\sigma} d \theta+A d g+n_{i}^{\sigma} d u_{i}=0
$$


J.F. Ganghoffer

as the surface counterpart of Gibbs-Duhem relation in classical thermodynamics [16].

The extension of the global energy balance (10) involving additional surface contributions then writes

$$
\begin{gathered}
\int_{\Omega_{g}} \omega_{v o l}[\theta, \mathbf{F}, \mu] d X_{g}+\int_{S_{g}} \omega_{\text {surf }}[\widetilde{\theta}, \widetilde{\mathbf{F}}, \widetilde{\mu}] d \sigma_{g} \\
=\int_{\Omega_{g}} \mathbf{f}_{0} \cdot \psi d X_{g}+\int_{\partial \Omega_{g}}(\mathbf{T} \cdot \mathbf{N}) \cdot \widetilde{\psi} d \sigma_{g} \\
+\int_{\partial \Omega_{g}}\left(\widetilde{\mathbf{T}}^{e l} \cdot \mathbf{N}\right) \cdot \widetilde{\psi} d \sigma_{g}+\int_{S_{g}} \mathbf{F}_{S} \cdot \widetilde{\psi} d \sigma_{g} \\
=\int_{\Omega_{g}} \mathbf{f}_{0} \cdot \psi d X_{g}+\int_{S_{g T}}(\mathbf{T} \cdot \mathbf{N})^{d} \cdot \widetilde{\psi} d \sigma_{g} \\
+\int_{S_{g \psi}}(\mathbf{T} \cdot \mathbf{N}) \cdot \widetilde{\psi}^{d} d \sigma_{g}+\int_{S_{g}} \mathbf{F}_{S} \cdot \widetilde{\psi} d \sigma_{g} \\
+\int_{\partial S_{g}}\left(\widetilde{\mathbf{T}}^{e l} \cdot \nu_{g}\right) \cdot \widetilde{\psi} d \sigma_{g},
\end{gathered}
$$

whereby a partition of the boundary has been made according to the nature of the boundary conditions (tractions $(\mathbf{T} . \mathbf{N})^{d}$ imposed on $S_{g t}$; position imposed on $S_{g \psi}$, with $\left.S_{g t} \cup S_{g \psi}=\partial \Omega_{g}, S_{g t} \cap S_{g \psi}=\emptyset\right)$. The surface and line forces, respectively $\mathbf{F}_{S}$, and $\widetilde{\mathbf{T}} . \nu_{g}$ appear in (22) as dual (in the sense of the work of external forces) to the position variables $\widetilde{\mathbf{x}}, \widetilde{\widetilde{\mathbf{x}}}, \widetilde{\widetilde{\mathbf{x}}}$ respectively, using the notations of the introductory section.

Based on the last equality in (22), a zero potential $Z[\theta, \mathbf{F}, \mu]$ is elaborated as

$$
\begin{gathered}
Z[\theta, \mathbf{F}, \mu]:=\int_{\Omega_{g}} \omega_{v o l}[\theta, \mathbf{F}, \mu] d X_{g} \\
+\int_{S_{g}} \omega_{\text {surf }}[\widetilde{\theta}, \widetilde{\mathbf{F}}, \widetilde{\mu}] d \sigma_{g}-\int_{\Omega_{g}} \mathbf{f}_{0} \cdot \psi d X_{g} \\
-\int_{S_{g T}}(\mathbf{T} \cdot \mathbf{N})^{d} \cdot \widetilde{\psi} d \sigma_{g}-\int_{S_{g \psi}}(\mathbf{T} \cdot \mathbf{N}) \cdot \widetilde{\psi}^{d} d \sigma_{g} \\
-\int_{S_{g}} \mathbf{f}_{S} \cdot \widetilde{\psi} d \sigma_{g}-\int_{\partial S_{g}}\left(\widetilde{\mathbf{T}} \cdot \nu_{g}\right) \cdot \widetilde{\widetilde{\psi}} d \sigma_{g} \equiv 0 .
\end{gathered}
$$

Since the potential $Z[\theta, \mathbf{F}, \mu]$ always vanishes, it is naturally endowed with an extremality property

$$
\delta Z[\theta, \mathbf{F}, \mu]=0
$$

The variation of $Z[\theta, \mathbf{F}, \mu]$ writes after a few transformations involving the surface divergence theorem as (dropping here the arguments of the volumetric and surface densities for simplicity)

$$
\begin{gathered}
\delta Z[\theta, \mathbf{F}, \mu]=\int_{\Omega_{g}}\left(-s \nabla \theta-n_{k} \partial_{n_{k}} \mu_{k} \nabla n_{k}+\mathbf{f}_{0}\right) \cdot \delta \mathbf{X}_{g} d X_{g} \\
+\int_{S_{g}}\left[\left(-s^{\sigma} \nabla_{S} \theta-n_{k}^{\sigma} \partial_{n_{k}^{\sigma}} \mu_{k} \nabla_{S} n_{k}^{\sigma}\right) \mathbf{I}+\nabla_{S} \cdot \widetilde{\mathbf{\Sigma}}\right. \\
\left.-\mathbf{P} . \mathbf{K}^{t} \cdot \partial_{N} \omega_{\text {surf }}+\mathbf{N} \cdot \mathbf{\Sigma}\right] \cdot \delta \mathbf{X}_{S} d \sigma_{g} \\
+\int_{\partial \Omega_{g}} \omega_{v o l} \mathbf{N} \cdot \delta \mathbf{X}_{S} d x_{g}-\int_{S_{g}} \mathbf{f}_{S} \cdot \delta \mathbf{X}_{S} d \sigma_{g} \\
-\int_{S_{g t}}(\mathbf{T} \cdot \mathbf{N})^{d} \cdot \delta \mathbf{X}_{S} d \sigma_{g}-\int_{\partial S_{g}}\left(\widetilde{\mathbf{T}} \cdot \nu_{g}\right) \cdot \delta \widetilde{\widetilde{\mathbf{X}}}_{S} d \sigma_{g} \\
+\int_{\partial S_{g}} \omega_{\text {surf }} \delta \widetilde{\widetilde{\mathbf{X}}}_{S} \cdot \nu_{g} d l_{g} \equiv 0
\end{gathered}
$$

assuming a nil prescribed position field and neglecting the variation of the surface stresses (the applied loads have been considered as dead loads). The equilibrium equations in strong form are obtained as a result of the arbitrariness and independence of the variations $\delta \mathbf{x}_{g}, \delta \mathbf{X}_{S}$ in (25):

$$
\begin{gathered}
-s \nabla \theta-n_{k} \partial_{n_{k}} \mu_{k} \nabla n_{k}+\nabla \cdot \boldsymbol{\Sigma}+\mathbf{f}_{0}=\mathbf{0} \quad \text { in } \Omega_{g}, \\
\left(\omega_{v o l}-s^{\sigma} \nabla_{S} \theta-n_{k}^{\sigma} \partial_{n_{k}^{\sigma}} \mu_{k} \nabla_{S} n_{k}^{\sigma}\right) \mathbf{N} \\
+\nabla_{S} \cdot \widetilde{\boldsymbol{\Sigma}}-\mathbf{P} . \mathbf{K}^{t} . \partial_{N} \omega_{\text {surf }}+\mathbf{f}_{S}=\mathbf{0} \quad \text { on } \quad S_{g} \backslash S_{g t}
\end{gathered}
$$

with boundary conditions

$$
\begin{gathered}
\left(-s^{\sigma} \nabla_{S} \theta-n_{k}^{\sigma} \partial_{n_{k}^{\sigma}} \mu_{k} \nabla_{S} n_{k}^{\sigma}\right)+\nabla_{S} \cdot \widetilde{\boldsymbol{\Sigma}} \\
-\mathbf{P} \cdot \mathbf{K}^{t} \cdot \partial_{N} \omega_{\text {surf }}+\omega_{v o l} \mathbf{N}=(\mathbf{T} \cdot \mathbf{N})^{d}, \\
\omega_{\text {surf }} \nu_{g}=\mathbf{T} \cdot \nu_{g} \Rightarrow \omega_{\text {surf }}=\nu_{g} \cdot \mathbf{T} \cdot \nu_{g} \text { on } \partial S_{g} .
\end{gathered}
$$

The factors of the variations $\delta \mathbf{x}_{g}$ and $\delta \mathbf{X}_{S}$ in (25) may be identified as material driving forces for growth (in the sense of configurational mechanics [17], since those variations represent virtual growth velocities; focusing on surface growth, the material surface driving force is then identified from (25) as

$$
\begin{gathered}
\widetilde{\Upsilon}=\left(-s^{\sigma} \nabla_{S} \theta-n_{k}^{\sigma} \partial_{n_{k}^{\sigma}} \mu_{k} \nabla_{S} n_{k}^{\sigma}\right) \mathbf{I}_{S} \\
+\nabla_{S} \cdot \widetilde{\boldsymbol{\Sigma}}-\mathbf{P} . \mathbf{K}^{t} \cdot \partial_{N} \omega_{\text {surf }}-\mathbf{f}_{S}+\omega_{\text {vol }} \mathbf{N} .
\end{gathered}
$$

As only the normal contribution can be considered as the true driving force, calculations similar to [24] lead to the normal projection of $\widetilde{\Upsilon}$ as

$$
\begin{aligned}
\widetilde{\Upsilon}_{g N}:= & \widetilde{\Upsilon} \cdot \mathbf{N}=\mathbf{N} \cdot \boldsymbol{\Sigma} \cdot \mathbf{N}+\widetilde{\boldsymbol{\Sigma}}: \mathbf{K}+\nabla_{S} \cdot(\widetilde{\boldsymbol{\Sigma}} \cdot \mathbf{N}) \\
& -\left(s^{\sigma} \nabla_{S} \theta+n_{k}^{\sigma} \partial_{n_{k}^{\sigma}} \mu_{k} \nabla_{S} n_{k}^{\sigma}\right)
\end{aligned}
$$

with the volumetric and surface Eshelby stress and curvature tensor given successively by

$$
\begin{gathered}
\boldsymbol{\Sigma}:=\mathbf{F}^{t} . \mathbf{T}-\omega_{\text {vol }}[\theta, \mathbf{F}, \mu] \mathbf{I} \\
\widetilde{\boldsymbol{\Sigma}}:=\widetilde{\mathbf{F}}^{t} . \widetilde{\mathbf{T}}-\omega_{\text {surf }}[\widetilde{\theta}, \widetilde{\mathbf{F}}, \widetilde{\mu}] \mathbf{I}_{S} ; \mathbf{K}:=-\nabla_{R} \mathbf{N} .
\end{gathered}
$$

It is important to note that both the volumetric and surface densities, respectively the scalar functions $\omega_{v o l}, \omega_{\text {surf }}$, account for all forms of energies (mechanical, thermal and 
chemical), as reflected in the right hand side of (6) and (19). Especially, the surface driving force in (30) incorporates curvature effects (first term), a mechanical driving force (second and third term, also partly included in the bulk contribution), and a thermal and chemical contribution (part of $\omega_{v o l}$ and $\omega_{\text {surf }}$, and last parenthesis).

\section{Conclusions}

General extreme principles have been established for continuum solid bodies undergoing simultaneously volumetric and surface growth, under the umbrella of open systems thermodynamics, resulting in volumetric and surface growth models when the stationnarity conditions are expressed. The resulting balance laws, Eqs. (16) through (18) for volumetric growth and (26)-(29) for surface growth, highlight the material driving forces for growth, based on volumetric and surface Eshelby stresses, respectively the normal projection of Eshelby stress identified in (17) and (30). This general framework opens the way for modeling situations of combined surface and volumetric growth, or volumetric growth per se with an additional surface energy contribution. The balance laws for the volumetric and surface Eshelby like stresses form the basis for the writing of evolution laws for the kinematic variables associated to growth phenomena.

Further work considering growth models within the framework of topology optimization is also under progress.

\section{REFERENCES}

[1] E.K. Rodriguez, A. Hoger, and A.D. McCullogh. "Stressdependent finite growth in soft elastic tissues", J. Biomechanics 27 (4), 455-46 (1994).

[2] M. Epstein and G.A. Maugin, "Thermomechanics of volumetric growth in uniform bodies", Int. J. Plasticity 16, 951-978 (2000).

[3] D. Ambrosi and F. Mollica, "On the mechanics of a growing tumor", Int. J Engng Sci. 40, 1297-1316 (2002).

[4] V.A. Lubarda and A. Hoger, "On the mechanics of solids with a growing mass", Int. J. Solids Struct. 39, 4627-4664 (2002).

[5] L. Taber and J.D. Humphrey, "Stress-modulated growth, remodeling and morphogenesis", Appl. Mech. Rev. 48, 487-545 (2001).

[6] K. Garikipati, H. Narayanan, E.M. Arruda, K. Grosh, and A. Calve, "Material forces in the context of biotissue remodeling", Proc. EUROMECH Colloquium 445 Mechanics of material forces $1, \mathrm{CD}-\mathrm{ROM}$ (2003).

[7] K.R. Rajagopal, "Multiple natural configurations in continuum mechanics", Rep. Inst. Comput. Appl. Mech. 6, CD-ROM (1995).
[8] K.R. Rajagopal and A.R. Srinivasa, "Mechanics of the inelastic behavior of materials. Part I: theoretical underpinnings", Int. J. Plast. 14, 945-967 (1998).

[9] J.D. Humphrey and K.R. Rajagopal, Math. Meth. Mod. App. Sci. 12 (3), 407-430 (2002).

[10] J.F Ganghoffer and B. Haussy, "Mechanical modeling of growth considering domain variation. Part I: constitutive framework, Int. J. Solids Struct. 42 (15), 4311-4337 (2005).

[11] G.A. Ateshian, "On the theory of reactive mixtures for modeling biological growth", Biomechan. Model. Mechanobiol. 6, 423-445 (2007).

[12] H. Narayanan, E.M. Arruda, K.Grosh, and K. Garikipati, "The micromechanics of fluid-solid interactions during growth in porous soft biological tissue", Biomech Model Mechanobiol, 8, 167-181 (2009).

[13] A. Di Carlo, "Surface and bulk growth unified", in: Mechanics of Material Forces. Advances in Mechanics and Mathematic, P. Steinmann and G.A. Maugin (eds.), vol. 11, Springer, Berlin, 2005.

[14] J.F. Ganghoffer, "A contribution to the mechanics and thermodynamics of surface growth. Application to bone remodeling", Int. J. Engng Sci. 1, doi:10.1016/j.ijengsci.2011.08.004 (2011).

[15] J.D. Goodstein. States of Matter, Dover Phoenix Editions, London, 1975.

[16] H.B. Callen. Thermodynamics and an Introduction to Thermostatics, J. Wiley, New York, 1985.

[17] G.A. Maugin. Material Inhomogeneities in elasticit, Chapman et al., London, 1993.

[18] G.A. Maugin, "On canonical equations of continuum thermomechanics", Mech. Res. Comm. 33, 705-710 (2006a).

[19] G.A. Maugin, "On the thermomechanics of continuous media with diffusion and /or weak nonlocality", Arch. Appl. Mech. 75, 723-738 (2006b).

[20] C.H. Wu, "The role of Eshelby stress in composition-generated and stress-assisted diffusion”, J. Mech. Phys. Solids 49 (8), 1771-1794 (2001).

[21] C.H. Wu, "Chemical potential and energy momentum tensor in single phase mixtures", Mechanics Research Communications 29, 493-499 (2002).

[22] J.D. Eshelby, "Energy relations and the energy-momentum tensor in continuum mechanics", in: Inelastic Behaviour of Solids, M.F. Kanninen, W.F. Adler, A.R. Rosenfield, and R.I. Jaffee (eds.), pp. 77-115, McGraw-Hill, New York, 1970.

[23] J.F. Ganghoffer, "On Eshelby tensors in the context of open systems: application to volumetric growth”, Int. J. Engng Sci. 1, doi: 10.1016/j.ijengsci.2010.04.003 (2010).

[24] J.F. Ganghoffer, "Mechanical modeling of growth considering domain variation - Part II: volumetric and surface growth involving Eshelby tensors", J. Mech. Phys. Solids 1, doi: 10.1016/j/jmps/2010.05.003 (2010). 